\title{
An improved optimization technique for estimation of solar photovoltaic parameters
}

\author{
Derick. $\mathrm{M}^{\mathrm{a}}$, Rani. $\mathrm{C}^{\mathrm{a}}{ }^{*}$, Rajesh. $\mathrm{M}^{\mathrm{a}}$, Farrag. M. $\mathrm{E}^{\mathrm{b}}$, Wang. $\mathrm{Y}^{\mathrm{c}}$ and Busawon. $\mathrm{K}^{\mathrm{c}}$ \\ ${ }^{a}$ School of Electrical Engineering, VIT University, Tamil Nadu, India \\ ${ }^{b}$ School of Engineering and Built Environment, Glasgow Caledonian University, Glasgow, UK \\ ${ }^{c}$ Faculty of Engineering and Environment, Northumbria University, Newcastle upon Tyne, UK
}

\section{ARTICLE INFO}

\section{Article history:}

Received

Received in revised form

Accepted

Available online

Keywords:

Double diode model

Genetic Algorithm

Pattern Search

Simulated Annealing

Wind Driven Optimization

\begin{abstract}
The nonlinear current vs voltage (I-V) characteristics of solar PV make its modelling difficult. Optimization techniques are the best tool for identifying the parameters of nonlinear models. Even though, there are different optimization techniques used for parameter estimation of solar $\mathrm{PV}$, still the best optimized results are not achieved to date. In this paper, Wind Driven Optimization (WDO) technique is proposed as the new method for identifying the parameters of solar PV. The accuracy and convergence time of the proposed method is compared with results of Pattern Search (PS), Genetic Algorithm (GA), and Simulated Annealing (SA) for single diode and double diode models of solar PV. Furthermore, for performance validation, the parameters obtained through WDO are compared with hybrid Bee Pollinator Flower Pollination Algorithm (BPFPA), Flower Pollination Algorithm (FPA), Generalized Oppositional Teaching Learning Based Optimization (GOTLBO), Artificial Bee Swarm Optimization (ABSO), and Harmony Search (HS). The obtained results clearly reveal that WDO algorithm can provide accurate optimized values with less number of iterations at different environmental conditions. Therefore, the WDO can be recommended as the best optimization algorithm for parameter estimation of solar PV.
\end{abstract}

\section{Introduction}

Today, due to the necessity of clean energy sources, the application of renewable and inexhaustible energy sources is gradually increasing. Among them, solar energy source seems to constitutes one of the best alternative solutions for energy provision. However, for this purpose, precise modeling of solar $\mathrm{PV}$ is required as it must be employ to predict the characteristic curves of solar PV at different weather condition of a particular area. This, in turn, is necessary for designing the corresponding inverter with high efficiency that is suitable for the given location. In addition to that, it will be beneficial to identify any mismatch in the PV array due to dust in the solar PV module by calculating the difference in real power generated by the module and predicted power by the model. This will enable to carry out maintenance at the right time [1].

The modeling of solar PV is generally derived using equivalent diode models. The current vs voltage (I-V) characteristics of solar PVs are mostly described using two types of diode models: single diode model and double diode model [2-4]. In this work, we focus on both single diode and double diode models of the solar PV. The parameters of the solar PV model vary with temperature and irradiance. Hence, precise estimation of the parameters is required to model the solar PV accurately. The popular approaches employed for parameter estimations are broadly categorized as analytical techniques [5], numerical extraction [6-9] and evolutionary algorithm techniques [10-14].

In the analytical technique, mathematical equations are used to find the parameters. Most of the values in the equations are not provided in the manufacturer datasheet. As a result, this method is not deemed accurate [4]. Numerical extraction technique is based on curve fitting. However, the application of curve fitting to the nonlinear equation of diode is quite difficult. Consequently, numerical extraction approach is not so popular either [8]. On the other hand, artificial intelligence techniques [11] are considered as excellent in dealing with nonlinear equations. In recent years, different optimization techniques have been introduced to estimate the parameters of solar PV; namely, the Genetic Algorithm (GA) [10], [12], Pattern Search (PS) optimization [13], Artificial Immune System (AIS) [15], Bacterial Foraging Algorithm (BFA) [16], Simulated Annealing (SA) [17],

* Corresponding author at. School of Electrical Engineering, VIT University, Vellore, Tamil Nadu, India;

E-mail addresses: derick.mathew@vit.ac.in (Derick. M), crani@vit.ac.in (Rani. C), mrajeshkumar@ vit.ac.in (Rajesh. M), mohamed.farrag@guc.ac.uk (Mohamed Emad. F), ywe5.wang@northumbria.ac.uk (Wang. Y), krishna.busawon@northumbria.ac.uk (Busawon. K) 
Differential Evaluation (DE) [18], Mutative-scale Parallel Chaos Optimization (MPCOA) [19], Harmony Search (HS) based algorithm [20], Artificial Bee Swarm Optimization (ABSO) algorithm [21], Artificial Bee Colony (ABSO) optimization [22], Flower Pollination Algorithm (FPA) [23], Levenberb - Marquard Algorithm with Simulated Annealing (LMSA) [24], Cuckoo Search (CS) [25], hybrid Bee Pollinator Flower Pollination Algorithm (BPFPA) [26], Fireworks Algorithm (FA) and Generalized Oppositional Teaching Learning Based Optimization (GOTLBO) [27]. However, these algorithms still require some modifications to find the most optimized parameter for different solar PV modules [23]. The most efficient algorithm for finding the optimized value of solar PV parameters are yet to be found.

In this work, we proposed Wind Driven Optimization (WDO) algorithm to optimize parameters of a single diode and double diode models of solar PV. The idea of WDO is developed by Zikri Bayraktar for electromagnetic application [28]. It is a population based heuristic global optimization technique for multidimensional problems. The algorithm contains four constants. Optimized values of these constants are generated using Covariance Matrix Adaptation Evolution Strategy (CMAES) technique [29].

The accuracy of the proposed optimization technique is measured using the value of Root Mean Square Error (RMSE). Convergence time is evaluated by the time required for the proposed method to reach the optimized value. In order to display the potential of the WDO algorithm, its accuracy and convergence time is compared with PS, GA, and SA available in the MATLAB optimization toolbox. In addition, the parameters obtained through WDO is compared with results obtained in recent literature like BPFPA [26], FPA [23], ABSO [21], HS [20]. All these investigations provide an evaluation on the accuracy and time of convergence of the proposed algorithm for parameter estimation of solar PV.

An outline of the paper is as follows: The mathematical modeling of solar PV is presented in next section. Section 3 presents the problem formulation. The WDO is explained in detail for solar PV parameter estimation in Section 4. This is followed by the discussion of results in Section 5. Finally, conclusions are presented in Section 6.

\section{Mathematical Modelling}

Many models have been proposed and developed by several researchers to estimate the solar PV parameters accurately [3]. Among them, the most popular and universally adopted models are the single diode and double diode models. In our work, both diode models are used to represent the behavior of solar PV module. In what follows, a description of both models is given.

\subsection{Single Diode Model}

Single diode model is commonly used to represent solar PV, because of its reduced complexity [31], [32]. The equivalent circuit of single diode model of solar PV is shown in Fig. 1.

By using Kirchhoff's current law (KCL), one can check that:

$$
I=I_{p h}-I_{D}-I_{p}
$$

Here, $I$ is solar PV current, $I_{p h}$ is the photon current generated by the incident light, $I_{D}$ is the diode current and $I_{p}$ is the current flowing through parallel resistance [32- 33].

$$
\begin{aligned}
& I=I_{p h}-I_{o}\left(\exp \left(\frac{V+I R_{S}}{N_{S} * a \frac{K T}{q}}\right)-1\right)-\frac{V+I R_{S}}{R p} \\
& I_{o}=\frac{I_{S C-S}+K_{I}\left(T-T_{S}\right)}{\exp \left(\frac{V_{O C-S}+K_{V}\left(T-T_{S}\right)}{N_{S} V_{t}}\right)-1} \\
& I_{p h}=\left(I_{p h-S}+K_{I}\left(T-T_{S}\right)\right) \frac{G}{G_{S}}
\end{aligned}
$$

Here $I_{o}$ is the reverse saturation current of diode, $V$ is solar PV voltage, $R_{p}$ is the parallel resistance, $R_{s}$ is the series resistance, $V_{O C-S}$ is the open circuit voltage at standard test condition, $K_{V}$ is open circuit voltage temperature coefficient, $N_{S}$ is the number of series cell per module, the temperature at standard test condition $T_{S}=25^{\circ} \mathrm{c}$, solar radiation at standard test condition $G_{S}=$ $1000 \mathrm{~W} / \mathrm{m}^{2}, K_{I}$ is the short circuit current temperature coefficient, $V_{t}$ is the thermal voltage of diode which depends on junction temperature and is given by:

$$
V_{t}=a \frac{K T}{q}
$$

where $a$ denotes the ideality factor of diode. $T$ expresses the junction temperature in Kelvin $(\mathrm{K}), q$ is the electron charge $\left(1.6021765 \times 10^{-19} \mathrm{C}\right)$ and $K$ is the Boltzmann constant $\left(1.38065 \times 10^{-23} \mathrm{~J} / K\right)$.

$I_{p h-S}$ is the photon current at standard test condition, and it is given by

$$
I_{p h-S}=I_{S C-S}\left(\frac{R_{P}+R_{S}}{R_{P}}\right)
$$

where $I_{S C-S}$ is the short circuit current at standard test conditions.

From Eq. (2), one can see that we require optimum values of five parameters $I_{p h}, I_{o}, R_{p}, R_{s}$, and $a$ in order to be able to generate the same I-V characteristic curve as obtained experimentally. Finally, it is important to note that Eq. 2 is an implicit equation in $I$.

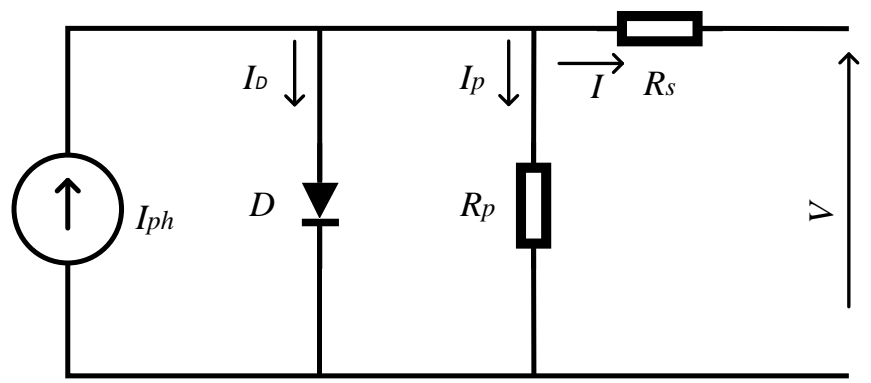

Fig. 1. Single - diode model of solar PV.

\subsection{Double Diode Model}

In double diode model, two diodes are connected in parallel to the photon current source. The second diode represents the recombination in the space charge region. The equivalent circuit of double diode model is shown in the Fig. 2.

By using KCL

$I=I_{p h}-I_{D 1}-I_{D 2}-I_{p}$

$I=I_{p h}-I_{o 1}\left(\exp \left(\frac{V+I R_{S}}{N_{S} V_{t 1}}\right)-1\right)-I_{o 2}\left(\exp \left(\frac{V+I R_{S}}{N_{S} V_{t 2}}\right)-1\right)-$

$\frac{V+I R_{S}}{R p}$ 
Here $I_{o 1}$ and $I_{o 2}$ are the reverse saturation currents and $V_{t 1}$ and $V_{t 2}$ are thermal voltages of diode 1 and diode 2 respectively. $I_{p h}$ can determined using the Eq. (4)

$$
\begin{aligned}
& V_{t 1}=a_{1} \frac{K T}{q} \\
& V_{t 2}=a_{2} \frac{K T}{q}
\end{aligned}
$$

where $a_{1}$ and $a_{2}$ denotes the ideality factor of diode 1 and diode 2 respectively.

From Eq. (8), it is clear that it is necessary to obtain optimized values of the seven parameters $I_{p h}, I_{o 1}, I_{o 2}, R p, R_{s}, a_{1}$ and $a_{2}$ in order to have an accurate double diode model of solar PV.

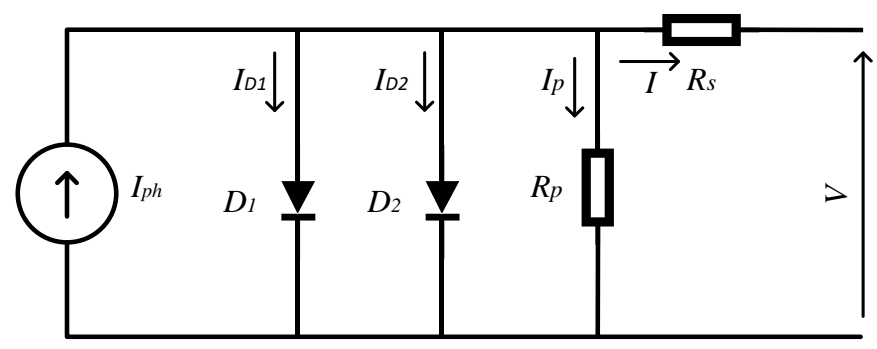

Fig. 2. Double - diode model of solar PV.

\section{Problem Formulation}

Any solar PV module can be modeled by using the single diode or double diode models. The main objective of this modeling is to enable the solar PV model to predict the I-V characteristics of the $\mathrm{PV}$ module. In order to minimize the error between predicted and actual I-V characteristics of PV module, one has to find the optimized parameters of the solar PV model. This can be done by using optimization algorithms.

As mentioned above single diode and double diode models of solar PV model have five and seven parameters respectively. In this paper, the values of resistances $R_{p}, R_{s}$ and ideality factor $a$ are determined using the proposed algorithm where as $I_{p h}$ and $I_{o}$ using Eqs. (3) and (4) in order to reduce the computational complexity.

The objective function is Root Mean Square Error (RMSE) between the measured and estimated current. The objective function will aggregate the absolute error and gives the measure of predictive power. The absolute difference between measured and estimated output current is the Individual Absolute Error (IAE). The error function of a single diode and double diode model is given in Eq. (11) and Eq. (12) respectively. The Sum of Squared Error (SSE) function is given in Eq. (13).

$$
f_{s}\left(V_{(m)}, I_{(m)} X\right)=I A E=\operatorname{abs}\left(I_{(m)}-\left(I_{p h}-I_{D(m)}-I_{p(m)}\right)\right)
$$

$f_{d}\left(V_{(m)}, I_{(m)}, X\right)=I A E=\operatorname{abs}\left(I_{(m)}-\left(I_{p h}-I_{D 1(m)}-I_{D 2(m)}-\right.\right.$

$\left.\left.I_{p(m)}\right)\right)$

$S S E=\sum_{i=1}^{N} I A E^{2}$

In Eq. (11) and Eq. (12) the vector $X$ represents the model parameters, for single and double diode model of solar PV respectively and $N$ is the number of experimental data.

The RMSE function is defined as follows:

$\mathrm{RMSE}=\sqrt{\frac{1}{N} S S E}$
The proposed WDO algorithm finds the optimized solar PV model parameter by minimizing the objective function.

\section{Wind Driven Optimization}

Wind driven optimization is a new nature inspired optimization technique [33]. The idea was developed by Zikri Bayraktar for electromagnetics application [28]. The motivation for WDO algorithm was based on the motion of microscopic air parcels in a multidimensional space. In earth's troposphere, the solar radiation varies based on the location. So, heating the surface of the earth varies according to the location, type of the region (water body, soil, cloudy), and rotation of earth [29]. The air pressure will be high at low-temperature area than high-temperature area. This difference in air pressure leads horizontal motion of air. The change in pressure is the pressure gradient [33], and is given as follows:

$\nabla P=\left(\frac{\partial P}{\partial x}, \frac{\partial P}{\partial y}, \frac{\partial P}{\partial z}\right)$

Here the air parcel is assumed to be dimensionless and weightless to reduce the computational complexity. Newton's second law states that total force $\left(F_{t}\right)$ applied on air parcel causes the air parcel to accelerate with an acceleration $a$ in the same direction of the force:

$\rho \cdot \vec{a}=\sum \overrightarrow{F_{t}}$

The four forces that create movement of air parcel are pressure gradient force $\left(F_{P G}\right)$, frictional force $\left(F_{F}\right)$, gravitational force $\left(F_{G}\right)$ and Coriolis force $\left(F_{C}\right)$.

Assuming that air has finite volume $(\delta \mathrm{V})$, the force due to pressure gradient can be expressed as Eq. 17. The friction force opposes the air parcel motion started by $F_{P G}$. The gravitational force pulls the air parcel to the center of the coordinate system from all dimensions. The rotation of the earth causes deflection in the motion of air parcel and named as Coriolis force. This force will work in such a way that velocity in one direction is influenced by velocity in another direction. All these forces can be expressed as:

$\overrightarrow{F_{P G}}=-\nabla P . \delta V$
$\overrightarrow{F_{F}}=-\rho \alpha \vec{u}$
$\overrightarrow{F_{G}}=\rho . \delta V \cdot g$
$\overrightarrow{F_{C}}=-2 \theta \times \vec{u}$

Here $\rho$ is the air density of a small air parcel, $\alpha$ is frictional coefficient, $\vec{u}$ wind velocity vector, $g$ is the gravitational constant, $\theta$ represents the rotation of earth.

So, by including $F_{P G}, F_{F}, F_{G}, F_{C}$ and ideal gas equation in total force Eq. 16, the latter can be rewritten as:

$\overrightarrow{\nabla u}=g+\left(-\nabla P \cdot \frac{R T}{P_{c u r}}\right)+(-\alpha \vec{u})+\left(-\frac{2 \theta \times \vec{u} R T}{P_{c u r}}\right)$

In Eq. 21 velocity of air parcel depends on pressure value. Consequently, if pressure value increases the velocity gets updated impractically. For that, Eq. 21 is modified based on the rank of the pressure. After every iteration, the air parcels are ranked in descending order based on their pressure values. If $i$ is the rank of the air parcel, velocity and position will be updated using the Eq. 22 and Eq. 23 respectively.

$$
\begin{aligned}
& \overrightarrow{u_{\text {new }}}=(1-\alpha) \vec{u}_{\text {cur }}-g x_{\text {cur }}+\left(| 1 - \frac { 1 } { i } | \cdot \left(x_{\text {opt }}-\right.\right. \\
& \left.\left.x_{\text {cur }}\right) R T\right)+\left(\frac{c \cdot \vec{u}_{\text {otherdirection }}}{i}\right) \\
& \overrightarrow{x_{\text {new }}}=\overrightarrow{x_{\text {old }}}+\overrightarrow{u_{\text {new }}}
\end{aligned}
$$


Here $\overrightarrow{u_{\text {new }}}$ is the velocity of next iteration, $\overrightarrow{u_{c u r}}$ is the velocity of current the iteration, $x$ is the position of the air parcel in search space, $x_{\text {opt }}$ is optimal position, $x_{\text {cur }}=$ current position, $c=$ $-2 R T$, and $\vec{u}_{\text {otherdirection }}=\overrightarrow{F_{C}}$.

In this parameter estimation problem, each dimension of an air parcel is the parameters of solar PV. So, in single diode model, the air parcel is in a three-dimensional space whereas, in the double diode model it is in four-dimensional space. The pressure of air parcels in a search space is evaluated using the objective function. Next, air parcels are ranked based on their objective function value. So, the velocity of air parcels is modified using their ranks and move to another position with that velocity. The air parcels continue their movement to find the lowest objective function value. The last step is to find the air parcel with lowest objective function value and their corresponding parameters.

For each dimension the WDO allows air parcel to travel in a bound of $[-1,1]$. The actual maximum and minimum limits of the problem are normalized to $[-1,1]$. To obtain the optimized objective function value, the coefficients $\alpha, g, R T, c$ in Eq. 22 play an important role. In order to find the optimized values of these constants Covariance Matrix Adaptation Evolution Strategy (CMAES) technique is used. It does not require any inputs other than population size [28]. Hence, CMAES is easy to implement for WDO application. The flow chart of wind the driven optimization algorithm is shown in Fig. 3.

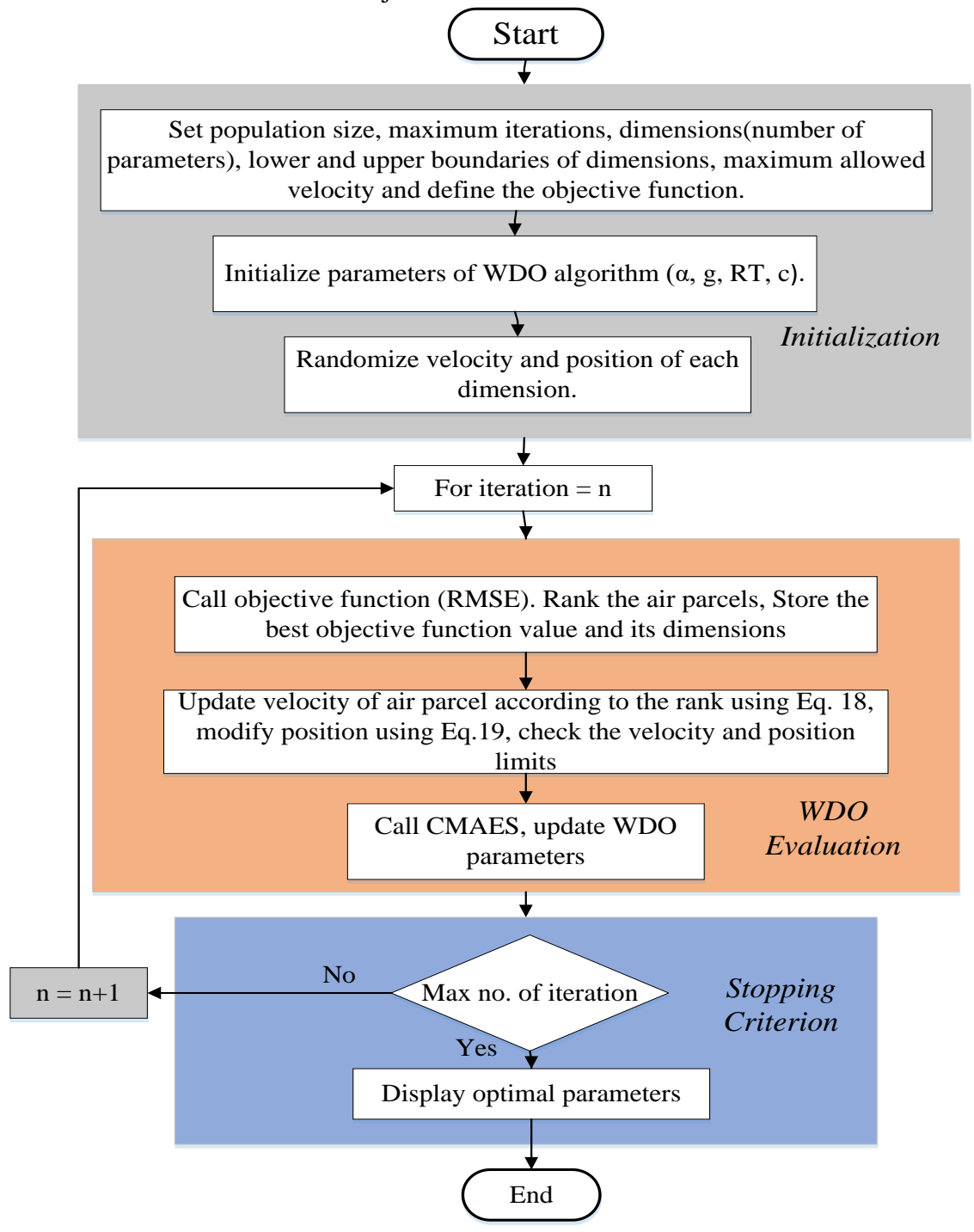

Fig. 3. Flowchart of Wind driven optimization

\section{Results and Discussion}

\subsection{Results of WDO is compared with PS, GA, and SA.}

Wind Driven Optimization algorithm is used to find the optimized parameters of a single diode and double diode solar model. In order to validate the accuracy of the proposed optimization algorithm, the result of WDO is compared with results obtained from PS, GA, and SA available in MATLAB optimization tool box.

Single diode and double diode models of solar PV is developed in MATLAB/Simulink to test the optimization techniques. The results obtained through PS, GA, and SA are compared with the proposed WDO algorithm results. The experimental data of multicrystal PV module Kyocera - KC200GT 215 given in [30] is used to find the objective function. The objective function is calculated based on the 18 set of experimental data. 
Here the ideality factor $a$ has a value between 1 and 2 . The value of series resistance $R_{S}$ is between $0.01 \Omega$ to $0.5 \Omega$, whereas the parallel resistance $R_{p}$ has value between $100 \Omega$ and $1000 \Omega$. The $I_{p h}$ and $I_{o}$ values are calculated using Eqs. (3) and (4). The data sheet values of Kyocera - KC200GT 215 module is given in Table 1 .

\subsubsection{Case study1: Single diode model.}

In this section, the validity of the proposed method is tested for single diode model. Table 2 indicates the values of $a$, $R_{s}, R_{p}, I_{p h}, I_{o}$ and RMSE WDO, PS, GA, and SA optimization techniques at standard test condition. It clearly exhibits that, the WDO gives very less RMSE value compared to other techniques. So, in terms of accuracy WDO is the best technique.

The Fig. 4 shows the convergence characteristics of four optimization techniques. From the fitness function curve, it is evident that the best fitness function value of 0.0008401 is obtained for WDO with less number of iterations. This clearly reveals that WDO algorithm performs well in terms of accuracy and computation time. The time required for the WDO to find the optimized values is $5.6 \mathrm{~ms}$ while PS and GA are $0.8 \mathrm{~ms}$ whereas SA required $0.02 \mathrm{~s}$.

The experimental data in [30] gives solar PV voltage and current at different irradiance and temperature values. Using the experimental data and WDO estimated data I-V characteristics of Kyocera - KC200GT 215 Solar PV module is plotted for $1000 \mathrm{~W} / \mathrm{m}^{2}, 800 \mathrm{~W} / \mathrm{m}^{2}, 600 \mathrm{~W} / \mathrm{m}^{2}, 400 \mathrm{~W} / \mathrm{m}^{2}$ and $200 \mathrm{~W} / \mathrm{m}^{2}$ in Fig. 5. Similarly, I-V characteristics for different temperature $25^{\circ} \mathrm{C}$, $50^{\circ} \mathrm{C}$ and $75^{\circ} \mathrm{C}$ is plotted in Fig. 6. Both Figures reflects the fact that the values estimated by the WDO algorithm give out accurate I-V characteristics which exactly replicate the experimental data.

\subsubsection{Case study2: Double diode model.}

In this section, double diode model is used to represent the solar PV. The optimized values of parameters such as $a_{1}, a_{2}, R_{s}, R_{p}$, and the derived values of $I_{p h}, I_{o}, \mathrm{RMSE}$ at standard test condition is presented in Table 3 . The I-V characteristic at different irradiance and temperature are plotted in Fig. 7 and Fig. 8 respectively.

Table 1

Datasheet values of Kyocera - KC200GT 215 module.

\begin{tabular}{lc} 
Maximum power $\left(P_{\max }\right)$ & $200 \mathrm{~W}(+10 \% /-5 \%)$ \\
$\begin{array}{l}\text { Voltage at maximum power point } \\
\left(V_{\text {mpp }}\right)\end{array}$ & $26.3 \mathrm{~V}$ \\
$\begin{array}{l}\text { Current at maximum power point } \\
\left(I_{m p p}\right)\end{array}$ & $7.61 \mathrm{~A}$ \\
Open circuit voltage $\left(V_{O C}\right)$ & $32.9 \mathrm{~V}$ \\
Short circuit current $\left(I_{S C}\right)$ & $8.21 \mathrm{~A}$ \\
Temperature coefficient of $V_{O C}$ & $-1.23 \times 10^{-1}$ \\
Temperature Coefficient of $I_{S C}$ & $3.18 \times 10^{-3}$ \\
Number of cells $\left(N_{S}\right)$ & 54 \\
\hline
\end{tabular}

From I-V characteristics curves it is observable that the parameter values obtained through WDO produce the accurate curve with insignificant RMSE value for an entire range of voltage in all irradiance and temperature conditions. Hence, both these case studies clearly substantiate that, the WDO technique can generate more accurate results in all weather conditions with a minimal time of computation.
Table 2

Estimated single diode model parameters of Kyocera - KC200GT 215 module.

\begin{tabular}{ccccc}
\hline & WDO & PS & GA & SA \\
\hline$a$ & 1.4172 & 1.7 & 1.4819 & 1.6118 \\
$R_{s}(\Omega)$ & 0.1132 & 0.0339 & 0.1067 & 0.0796 \\
$R_{p}(\Omega)$ & 747.41 & 624.382 & 728.58 & 713.110 \\
$I_{p h}(A)$ & 8.1812 & 8.2104 & 8.2112 & 8.2109 \\
$I_{o}(\mu A)$ & 0.4423 & 7.1836 & 0.9220 & 3.3484 \\
RMSE & 0.00084 & 0.001796 & 0.00188 & 0.001875 \\
\hline
\end{tabular}

Table 3

Estimated double diode model parameters of Kyocera - KC200GT 215 module.

\begin{tabular}{ccccc} 
module. & WDO & PS & GA & SA \\
\hline$a_{1}$ & 1.9667 & 1.01 & 1.17 & 1.12 \\
$a_{2}$ & 1.5370 & 1.9 & 1.4324 & 1.5631 \\
$R_{s}(\Omega)$ & 0.99 & 0.031 & 0.0691 & 0.01783 \\
$R_{p}(\Omega)$ & 784.4062 & 793.215 & 763.3564 & 862.97 \\
$I_{p h}(A)$ & 8.1914 & 8.2107 & 8.2103 & 8.2102 \\
$I_{o 1}(A)$ & $4.746 \times 10^{-5}$ & $5.22 \times 10^{-10}$ & $1.29 \times 10^{-11}$ & $5.24 \times 10^{-9}$ \\
$I_{o 2}(A)$ & $1.632 \times 10^{-6}$ & $3.12 \times 10^{-10}$ & $5.3 \times 10^{-7}$ & $2.12 \times 10^{-9}$ \\
$\mathrm{RMSE}$ & 0.00106 & 0.0029 & 0.0029 & 0.003 \\
\hline
\end{tabular}

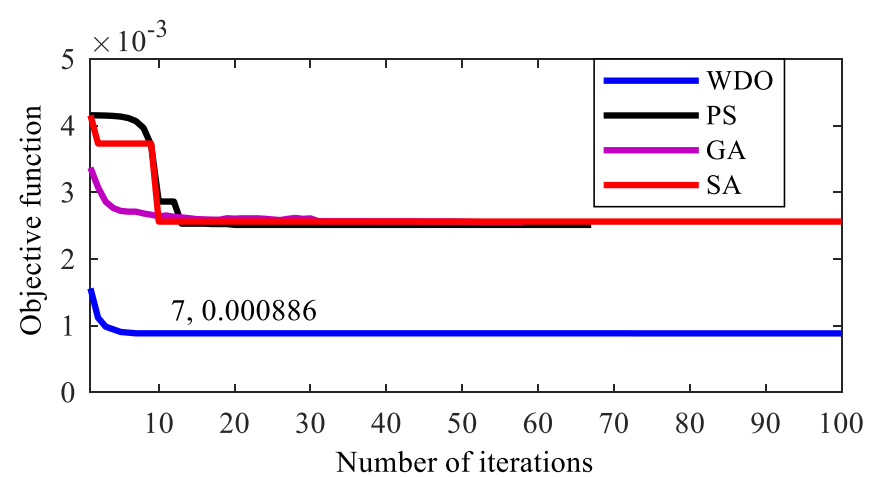

Fig. 4. Convergence characteristics of WDO, PS, GA, and SA.

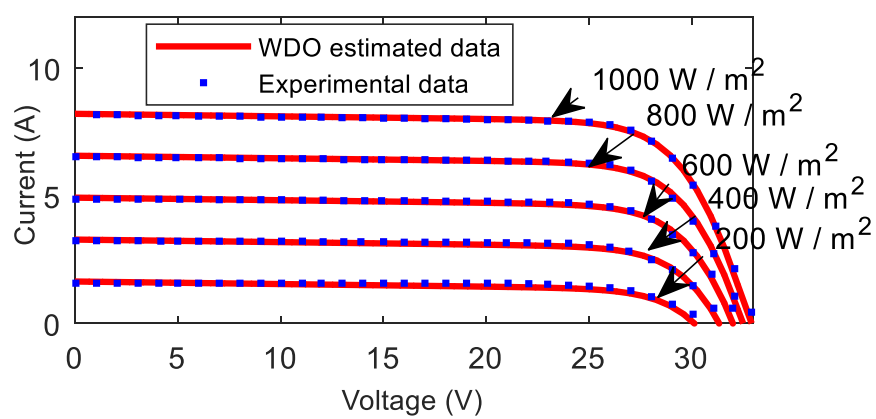

Fig. 5. Comparison of experimental data and WDO estimated data of Kyocera - KC200GT 215 module at different irradiance (single diode model).

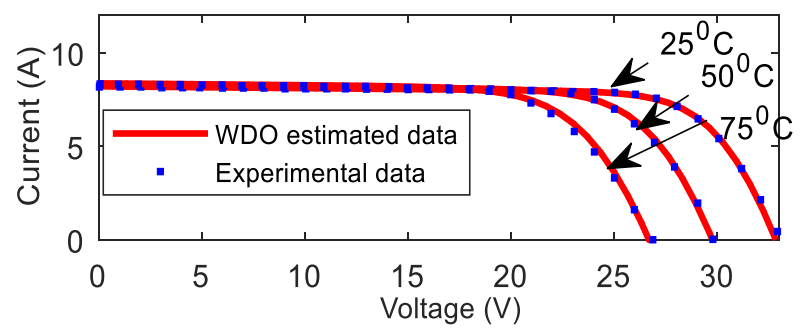

Fig. 6. Comparison of experimental data and WDO estimated data of Kyocera - KC200GT 215 module at different temperature (single diode model). 


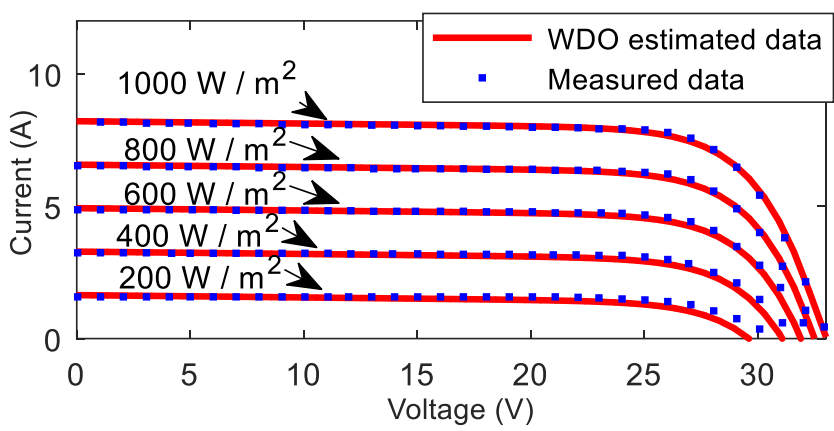

Fig. 7. Comparison of experimental data and WDO estimated data of Kyocera - KC200GT 215 module at different irradiance (double diode model).

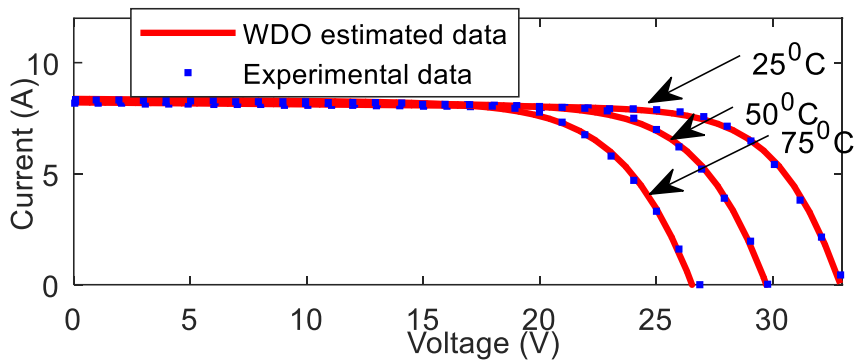

Fig. 8. Comparison of experimental data and WDO estimated data of Kyocera - KC200GT 215 module at different temperature (double diode model).

\subsection{Results of WDO is compared with recent literature.}

In order to further verify the performance of the WDO algorithm, the latter is examined with the experimental data of 57 $\mathrm{mm}$ dia RTC France silicon solar cell at $1000 \mathrm{~W} / \mathrm{m}^{2}$ irradiance and $33^{\circ} \mathrm{C}$ temperature presented in [34]. The parameters of a single diode and double diode model of the cell is estimated through WDO algorithm. The lower and upper boundaries of $a, R_{s}$, and $R_{p}$ are assigned as ( 1 to 2 ), ( 0.01 to 0.08$) \Omega$, and (25 to 75$) \Omega$ respectively. The optimized values of solar PV parameters along with RMSE for single diode and double diode models are presented in Table 4 and 5. It shows the comparison of results obtained through the optimization techniques presented in recent research papers such as BPFPA [26], Generalized Oppositional Teaching Learning Based Optimization (GOTLBO) [27], FPA [23], ABSO [21], HS [20] with WDO algorithm. From Table 4 and 5 it is clear that WDO algorithm provides the least RMSE value $\left(0.08664 \times 10^{-4}\right.$ and $\left.0.065237 \times 10^{-4}\right)$ while comparing with other optimization techniques.

The convergence curve of WDO algorithm for the single diode and double diode model is shown in Fig. 9 and 10 respectively. They prove that convergence time for WDO is very less. In parameter estimation of single diode model, WDO reaches a RMSE value of $8.664 \mathrm{e}-6$ after 100 iterations. Whereas, BPFPA and FPA took more than 500 iterations to obtain RMSE values of 8.456e-4 for same environmental conditions. Similarly, for double diode model, WDO algorithm reached a RMSE value of 6.5237e-6 after 750 iterations, whereas BPFPA and FPA reach $7.917 \mathrm{e}-4$ after 500 iterations.

In order to verify the accuracy of WDO determined parameter values, the $\mathrm{I}-\mathrm{V}$ characteristics at $1000 \mathrm{~W} / \mathrm{m} 2$ and $33^{\circ} \mathrm{C}$ for single diode model and double diode model of solar PV are plotted in Fig. 11 and 12 respectively. They clearly show that the I-V characteristic curve accurately replicates the experimental data provided in [34]. In order to validate the accuracy of curve fit between measured and estimated values, error analysis is used. So, Relative Error (RE) between measured and estimated values of PV current for the single diode and double diode models are calculated using the below mentioned formula.

$$
R E=\frac{I_{\text {measured }}-I_{\text {estimated }}}{I_{\text {measured }}}
$$

The RE value obtained using WDO for the single diode and double diode is compared with BPFPA [26], and HS [20] and tabulated in Table 6 and 7. From Tables, it is obvious that relative error for WDO is low while comparing the other optimization techniques considered for comparison.

Table 4

Comparison between estimated single diode model parameters of RTC France solar cell using WDO and other recent optimization techniques.

\begin{tabular}{ccccccc}
\hline & WDO & BPFPA [26] & GOTLBO [27] & FPA [23] & ABSO [21] & HS [20] \\
\hline$a$ & 1.4808 & 1.4774 & 1.48382 & 1.47707 & 1.47583 & 1.47538 \\
$R_{S}(\Omega)$ & 0.036768 & 0.03666 & 0.036265 & 0.0365466 & 0.03659 & 0.03663 \\
$R_{p}(\Omega)$ & 57.74614 & 57.7156 & 54.115426 & 52.8771 & 52.2903 & 53.5946 \\
$I_{p h}(A)$ & 0.7608 & 0.76 & 0.76078 & 0.76079 & 0.7608 & 0.7607 \\
$I_{o}(\mu A)$ & 0.3223 & 0.3106 & 0.3315 & 0.3106 & 0.3062 & 0.30495 \\
$\operatorname{RMSE}\left(\times 10^{-4}\right)$ & 0.08664 & 7.27 & 9.8744 & 7.7301 & 9.9124 & 9.951 \\
\hline
\end{tabular}

Table 5

Comparison between estimated double diode model parameters of RTC France solar cell using WDO and other recent optimization techniques.

\begin{tabular}{|c|c|c|c|c|c|c|}
\hline & WDO & BPFPA [26] & GOTLBO [27] & FPA [23] & ABSO [21] & HS [20] \\
\hline$a_{1}$ & 1.51162 & 1.4793 & 1.99973 & 1.4777 & 1.46512 & 1.49439 \\
\hline$a_{2}$ & 1.38434 & 2.00 & 1.448974 & 2 & 1.98152 & 1.49439 \\
\hline$R_{S}(\Omega)$ & 0.037433 & 0.0364 & 0.036783 & 0.0363342 & 0.03657 & 0.03545 \\
\hline$R_{p}(\Omega)$ & 52.6608 & 59.624 & 56.075304 & 52.3475 & 54.6219 & 46.82696 \\
\hline$I_{p h}(A)$ & 0.7606 & 0.7600 & 0.7607 & 0.760795 & 0.76078 & 0.76176 \\
\hline$I_{o 1}(\mu A)$ & 0.2531 & 0.3211 & 0.800195 & 0.3008 & 0.26713 & 0.12545 \\
\hline$I_{o 2}(\mu A)$ & 0.04827 & 0.04528 & 0.220462 & 0.166157 & 0.38191 & 0.2547 \\
\hline $\operatorname{RMSE}\left(\times 10^{-4}\right)$ & 0.065237 & 7.23 & 9.83177 & 7.8425 & 9.8344 & 12.6 \\
\hline
\end{tabular}




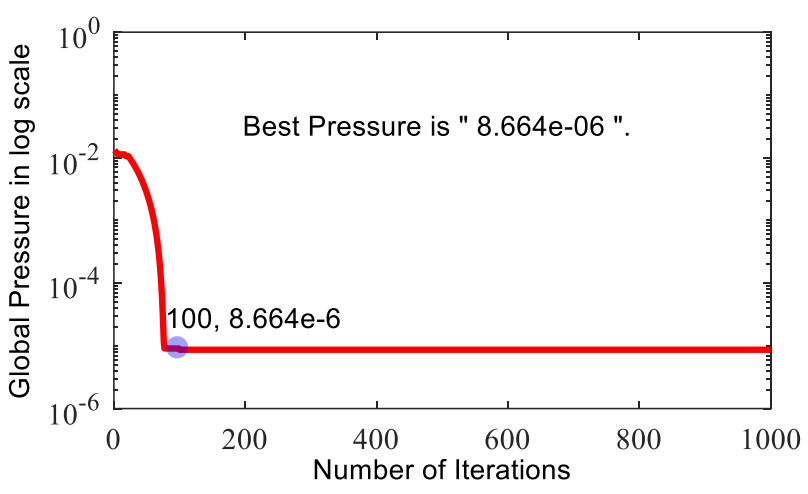

Fig. 9. Convergence curve of WDO algorithm for optimizing the parameters of single diode model

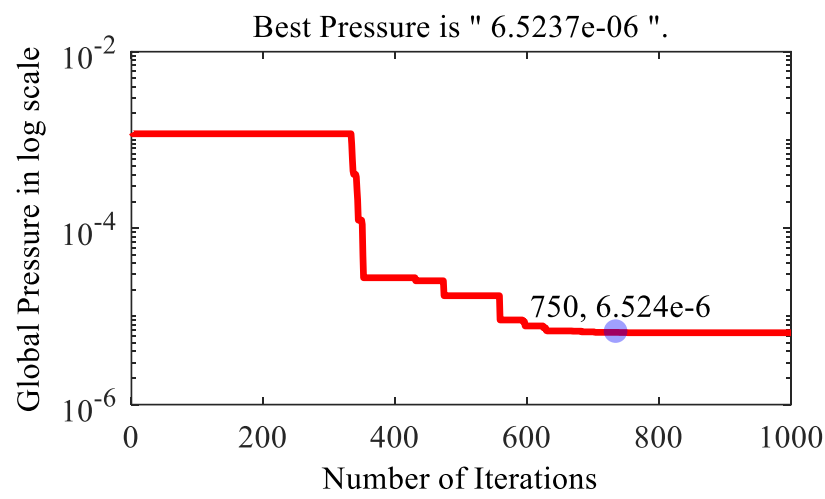

Fig. 10. Convergence curve of WDO algorithm for optimizing the parameters of double diode model

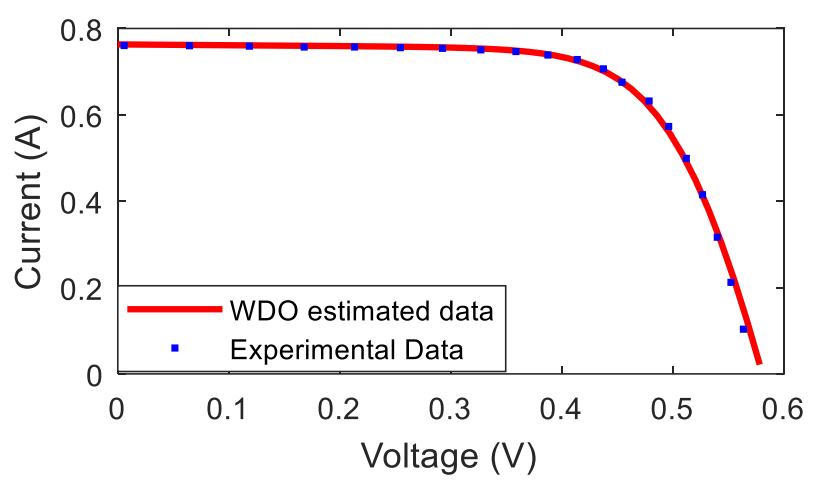

Fig. 11. Comparison of experimental data and WDO estimated data of RTC France solar cell at $1000 \mathrm{~W} / \mathrm{m}^{2}$ and $33^{\circ} \mathrm{C}$ for single diode model.

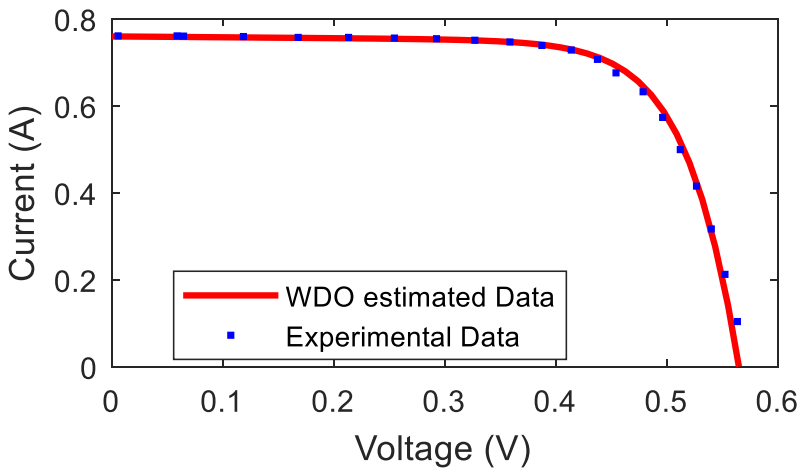

Fig. 12. Comparison of experimental data and WDO estimated data of RTC France solar cell at $1000 \mathrm{~W} / \mathrm{m}^{2}$ and $33^{\circ} \mathrm{C}$ for double diode model.

Table 6

The comparison of relative error values of WDO, BPFPA, HS for single diode model

\begin{tabular}{|c|c|c|c|c|c|c|c|c|}
\hline \multirow[t]{2}{*}{ Data } & \multirow[t]{2}{*}{ Vmeasured } & \multirow[t]{2}{*}{ Imeasured } & \multicolumn{2}{|c|}{ WDO } & \multicolumn{2}{|c|}{ BPFPA [26] } & \multicolumn{2}{|c|}{ HS [20] } \\
\hline & & & Iestimated & $\mathbf{R E}$ & Iestimated & $\mathrm{RE}$ & Iestimated & $\mathrm{RE}$ \\
\hline 1 & -0.2057 & 0.764 & 0.764 & 0.00016 & 0.764 & 0.00012 & 0.764 & -0.00036 \\
\hline 2 & -0.1291 & 0.762 & 0.763 & -0.00072 & 0.762 & 0.00032 & 0.762 & -0.00109 \\
\hline 3 & -0.0588 & 0.761 & 0.761 & -0.00109 & 0.761 & $-4.9 e-5$ & 0.761 & -0.00133 \\
\hline 4 & 0.0057 & 0.760 & 0.760 & 0.00037 & 0.759 & 0.00142 & 0.760 & 0.000272 \\
\hline 5 & 0.0646 & 0.760 & 0.759 & 0.00106 & 0.758 & 0.00210 & 0.759 & 0.001078 \\
\hline 6 & 0.1185 & 0.759 & 0.758 & 0.00098 & 0.757 & 0.00203 & 0.758 & 0.001113 \\
\hline 7 & 0.1678 & 0.757 & 0.757 & -0.00048 & 0.757 & 0.00057 & 0.757 & -0.00025 \\
\hline 8 & 0.2132 & 0.757 & 0.756 & 0.00069 & 0.755 & 0.00174 & 0.756 & 0.001015 \\
\hline 9 & 0.2545 & 0.755 & 0.755 & $4.08 e-5$ & 0.754 & 0.00108 & 0.755 & 0.000441 \\
\hline 10 & 0.2924 & 0.754 & 0.754 & -0.00011 & 0.753 & 0.00090 & 0.753 & 0.000353 \\
\hline 11 & 0.3209 & 0.750 & 0.751 & -0.0017 & 0.751 & -0.000 & 0.751 & -0.00126 \\
\hline 12 & 0.3585 & 0.746 & 0.747 & -0.0017 & 0.747 & 0.0008 & 0.747 & -0.0012 \\
\hline 13 & 0.3873 & 0.738 & 0.740 & -0.0028 & 0.739 & -0.0019 & 0.740 & -0.00221 \\
\hline 14 & 0.4137 & 0.728 & 0.727 & 0.0003 & 0.727 & 0.00090 & 0.727 & 0.000802 \\
\hline 15 & 0.4373 & 0.706 & 0.707 & -0.0010 & 0.706 & -0.0006 & 0.706 & -0.00066 \\
\hline 16 & 0.459 & 0.675 & 0.675 & 0.00024 & 0.675 & 0.00014 & 0.675 & 0.000293 \\
\hline 17 & 0.4784 & 0.632 & 0.630 & 0.00231 & 0.631 & 0.00150 & 0.630 & 0.001744 \\
\hline 18 & 0.490 & 0.573 & 0.571 & 0.0028 & 0.572 & 0.00126 & 0.572 & 0.001553 \\
\hline 19 & 0.5119 & 0.499 & 0.498 & 0.00051 & 0.499 & $0-0.0013$ & 0.499 & -0.00108 \\
\hline 20 & 0.5265 & 0.413 & 0.412 & 0.0011 & 0.413 & -0.00171 & 0.413 & -0.00134 \\
\hline 21 & 0.5398 & 0.316 & 0.316 & 0.00070 & 0.317 & -0.00302 & 0.317 & -0.00248 \\
\hline 22 & 0.5521 & 0.212 & 0.210 & 0.0047 & 0.212 & -0.00183 & 0.212 & -0.00076 \\
\hline 23 & 0.5633 & 0.103 & 0.102 & 0.01047 & 0.103 & 0.003957 & 0.102 & 0.007188 \\
\hline 24 & 0.5736 & -0.010 & -0.009 & 0.01 & -0.008 & 0.12410 & -0.009 & 0.075 \\
\hline 25 & 0.5833 & -0.123 & -0.124 & -0.00821 & -0.123 & -0.00596 & -0.124 & -0.01171 \\
\hline 26 & 0.59 & -0.210 & -0.209 & 0.003904 & -0.208 & 0.007587 & -0.209 & 0.003333 \\
\hline
\end{tabular}


Table 7

The comparison of relative error values of WDO, BPFPA, HS for double diode model

\begin{tabular}{|c|c|c|c|c|c|c|c|c|}
\hline \multirow[t]{2}{*}{ Data. } & \multirow[t]{2}{*}{ Vmeasured } & \multirow[t]{2}{*}{ Imeasured } & \multicolumn{2}{|c|}{ WDO } & \multicolumn{2}{|c|}{ BPFPA [26] } & \multicolumn{2}{|c|}{ HS [20] } \\
\hline & & & Iestimated & RE & Iestimated & $\mathrm{RE}$ & Iestimated & RE \\
\hline 1 & -0.2057 & 0.764 & 0.764 & $3.912 \mathrm{e}-5$ & 0.764 & $9.65 e-5$ & 0.764 & 0.00011 \\
\hline 2 & -0.1291 & 0.762 & 0.762 & -0.00067 & 0.762 & 0.000361 & 0.762 & 0.0007 \\
\hline 3 & -0.0588 & 0.761 & 0.761 & -0.00089 & 0.760 & $-3.9 e-5$ & 0.761 & 0.001 \\
\hline 4 & 0.0057 & 0.760 & 0.759 & 0.00071 & 0.759 & 0.0014 & 0.760 & 0.0004 \\
\hline 5 & 0.0646 & 0.760 & 0.758 & 0.00153 & 0.758 & 0.002 & 0.759 & 0.0011 \\
\hline 6 & 0.1185 & 0.759 & 0.757 & 0.001575 & 0.757 & 0.0019 & 0.758 & 0.00107 \\
\hline 7 & 0.1678 & 0.757 & 0.756 & 0.000214 & 0.756 & 0.0004 & 0.757 & 0.0003 \\
\hline 8 & 0.2132 & 0.757 & 0.755 & 0.00148 & 0.755 & 0.0016 & 0.756 & 0.0008 \\
\hline 9 & 0.2545 & 0.755 & 0.754 & 0.00089 & 0.754 & 0.00098 & 0.755 & 0.0003 \\
\hline 10 & 0.2924 & 0.754 & 0.753 & 0.00077 & 0.753 & 0.0008 & 0.753 & 0.0002 \\
\hline 11 & 0.3209 & 0.750 & 0.751 & -0.00094 & 0.751 & -0.0008 & 0.751 & 0.0012 \\
\hline 12 & 0.3585 & 0.746 & 0.747 & -0.00104 & 0.747 & -0.0008 & 0.747 & 0.0010 \\
\hline 13 & 0.3873 & 0.738 & 0.740 & -0.00233 & 0.739 & -0.0019 & 0.739 & 0.0019 \\
\hline 14 & 0.4137 & 0.728 & 0.727 & 0.00036 & 0.727 & 0.0010 & 0.727 & 0.0011 \\
\hline 15 & 0.4373 & 0.706 & 0.707 & -0.0015 & 0.706 & -0.0045 & 0.706 & 0.0002 \\
\hline 16 & 0.459 & 0.675 & 0.676 & -0.00095 & 0.675 & 0.0004 & 0.675 & 0.0005 \\
\hline 17 & 0.4784 & 0.632 & 0.631 & 0.00041 & 0.630 & 0.0016 & 0.630 & 0.00186 \\
\hline 18 & 0.490 & 0.573 & 0.572 & 0.00026 & 0.572 & 0.0016 & 0.572 & 0.00157 \\
\hline 19 & 0.5119 & 0.499 & 0.500 & -0.00257 & 0.499 & -0.0010 & 0.499 & 0.0011 \\
\hline 20 & 0.5265 & 0.413 & 0.413 & 0.00413 & 0.413 & -0.00137 & 0.413 & 0.0014 \\
\hline 21 & 0.5398 & 0.316 & 0.317 & -0.00283 & 0.317 & -0.00266 & 0.317 & 0.0024 \\
\hline 22 & 0.5521 & 0.212 & 0.211 & 0.00424 & 0.212 & -0.00126 & 0.212 & 0.0005 \\
\hline 23 & 0.5633 & 0.103 & 0.102 & 0.0073 & 0.102 & 0.00560 & 0.102 & 0.0078 \\
\hline 24 & 0.5736 & -0.010 & -0.009 & 0.0100 & -0.009 & 0.09727 & 0.009 & 0.0706 \\
\hline 25 & 0.5833 & -0.123 & -0.125 & -0.0020 & -0.124 & -0.00932 & 0.124 & 0.011 \\
\hline 26 & 0.59 & -0.210 & -0.208 & 0.0095 & -0.208 & 0.004979 & 0.209 & 0.0040 \\
\hline
\end{tabular}

\section{Conclusion}

Accurate modeling of solar PV is necessary before designing the entire PV system. The optimized parameter of single and double diode models plays an important role for accurate modeling. This paper presented a new Wind Driven Optimization algorithm for parameter estimation of solar PV. The performance of WDO algorithm was verified by comparing its results with PS, GA, and SA algorithms using MATLAB optimization tool box. Results of WDO clearly shows a better performance in terms of accuracy and convergence. In addition, in order to further validate the proposed algorithm, the WDO is compared with the optimization techniques presented in recent literature. Compared to recent optimization algorithms presented in literature such as hybrid Bee Pollinator Flower Pollination Algorithm, Flower Pollination Algorithm, Generalized Oppositional Teaching Learning Based Optimization, Artificial Bee Swarm Optimization, and Harmony Search WDO shows better results. As a result, WDO algorithm is recommended as the accurate and fastest optimization algorithm for parameter estimation of solar PV modules.

\section{References}

[1] Babu, T. Sudhakar, et al. "Parameter extraction of two diode solar PV model using Fireworks algorithm." Solar Energy 140 (2016): 265-276.

[2] Ghias Farivar, Behzad Asaei. "A New Approach for Solar Module Temperature Estimation Using the Simple Diode Model”. IEEE Trans Energy Conversion 2011; 26; 1118-1126.
[3] Barth, Nicolas, et al. "PV panel single and double diode models: Optimization of the parameters and temperature dependence." Solar Energy Materials and Solar Cells 148 (2016): 87-98.

[4] A. Rezaee Jordehi. "Parameter estimation of solar photovoltaic (PV) cells: A review". Renewable and Sustainable Energy Reviews. 2016; 61; 354-371.

[5] Daniel. S. H. Chan, Jacob. C. H. Phang. "Analytical Methods for the Extraction of Solar - Cell Single- and Double - Diode Model Parameter from I - V Characteristics". IEEE Trans Electronic Devices. 1987 ; 34 ; 286-293.

[6] P. Wolf, V. Benda. "Identification of PV solar cells and modules parameters by combining statical and analytical methods". Solar Energy. 2013; 93(0); 151-157.

[7] Kashif Ishaque, Zainal Salam, Hamed Taheri, Amir Shamsudin. "A critical evaluation of EA computation methods for photvoltaic all parameter extraction based on two diode model". Solar Energy. 2011; 85(9); 1768-1779. [8] Kashif Ishaque, Zainal Salam, Saad Mekhilef, Amir Shamsudin. "Parameter extraction of solar photo voltaic modules using penalty based differential evolution". Applied Energy. 2012; 99(0); 297-308.

[9] M. Barukcic, V.Corluka, K. Miklosevic. "The irradiance and temperature dependent mathematical model for estimation of photvoltaic panel performances". Energy Conversion and Management. 2015; 101; 229-238.

[10] M. S. Ismail, M. Moghavvemi, T. M. I. Mahila. "Characterization of PV panel and global optimization of its model parameter using genetic algorithm". Energy Conversion and Management. 2013; 73; 10-25.

[11] Jieming Ma, Ziqiang Bi, Tiew On Ting, Shiyuan Hao, Wanjun Hao. "Comparative performance on photvoltaic model parameter identification via bio-inspired algorithms”. Solar Energy. 2016; 132; 606-616.

[12] Moldovan. N, Picos, R., et al., 2009. "Parameter extraction of solar cell compact model using genetic algorithms", CDE 2009. Electron. Dev., Spanish Conference.

[13] Derick, M., et al. "Estimation of Solar Photovoltaic Parameters Using Pattern Search Algorithm." International Conference on Emerging Trends in Electrical, Electronic and Communications Engineering. Springer, Cham, 2016.

[14] Rajasekar, N., et al. "Application of modified particle swarm optimization for maximum power point tracking under partial shading condition." Energy Procedia 61 (2014): 2633-2639. 
[15] Xiaoping, Chen, Qu Bo, and Lu Gang. "An application of immune algorithm in FIR filter design." Neural Networks and Signal Processing, 2003. Proceedings of the 2003 International Conference on. Vol. 1. IEEE, 2003.

[16] N.Rajesekar, Neeraja Krishna Kumar, Rini Venugopalan. "Bacterial Foraging Algorithm based PV parameter estimation”. Solar Energy 2013; 97:255-265.

[17] K. M. El-Naggar, M. R. Al Rashidi, M. F. Al Hajri, A. K. Al-Othman. "Simulated annealing algorithm for photovoltaic parameter identification". Solar Energy. 2012; 86(1); 266-274.

[18] da Costa, Wagner Teixeira, et al. "Identification of photovoltaic model parameters by differential evolution." Industrial Technology (ICIT), 2010 IEEE International Conference on. IEEE, 2010.

[19] Yuan, Xiaofang, Yongzhong Xiang, and Yuqing He. "Parameter extraction of solar cell models using mutative-scale parallel chaos optimization algorithm." Solar Energy 108 (2014): 238-251.

[20] Askarzadeh, Alireza, and Alireza Rezazadeh. "Parameter identification for solar cell models using harmony search-based algorithms." Solar Energy 86.11 (2012): 3241-3249.

[21] Askarzadeh, Alireza, and Alireza Rezazadeh. "Artificial bee swarm optimization algorithm for parameters identification of solar cell models." Applied Energy 102 (2013): 943-949.

[22] Oliva, Diego, Erik Cuevas, and Gonzalo Pajares. "Parameter identification of solar cells using artificial bee colony optimization." Energy 72 (2014): 93-102.

[23] Alam, D. F., D. A. Yousri, and M. B. Eteiba. "Flower pollination algorithm based solar PV parameter estimation." Energy Conversion and Management 101 (2015): 410-422.

[24] Dkhichi, Fayrouz, et al. "Parameter identification of solar cell model using Levenberg-Marquardt algorithm combined with simulated annealing." Solar Energy 110 (2014): 781-788.

[25] Ma, Jieming, et al. "Parameter estimation of photovoltaic models via cuckoo search." Journal of Applied Mathematics 2013 (2013).

[26] Ram, J. Prasanth, et al. "A new hybrid bee pollinator flower pollination algorithm for solar PV parameter estimation." Energy Conversion and Management 135 (2017): 463-476.

[27] Chen, $\mathrm{Xu}$, et al. "Parameters identification of solar cell models using generalized oppositional teaching learning based optimization." Energy 99 (2016): 170-180.

[28] Bayraktar, Zikri, Muge Komurcu, and Douglas H. Werner. "Wind Driven Optimization (WDO): A novel nature-inspired optimization algorithm and its application to electromagnetics." 2010 IEEE Antennas and Propagation Society International Symposium. IEEE, 2010.

[29] Bhandari, Ashish Kumar, et al. "Cuckoo search algorithm and wind driven optimization based study of satellite image segmentation for multilevel thresholding using Kapur's entropy." Expert Systems with Applications 41.7 (2014): 3538-3560.

[30] Pauls, Catherine. Optimization approaches for parameter estimation and maximum power point tracking (MPPT) of photovoltaic systems. Diss. University of Liverpool, 2014.

[31] Marcelo Gradella. VIllalva, Jonas Rafael Gazoli, ERNESTO Ruppert Fiho. "Comprihensive Approach of Modelling and Simulation of Photovoltaic Array”. IEEE Trans Power Electronics. 2009; 24; 1198-1208.

[32] Abir Chatterjee, Ali Keyhani, Dhruv Kapoor. "Identification of Photovoltaic Source Modules". IEEE Trans Energy Conversion. 2011; 26; 883-889.

[33] Bayraktar, Zikri. Novel meta-surface design synthesis via nature-inspired optimization algorithms. Diss. The Pennsylvania State University, 2011.

[34] Easwarakhanthan T, Bottin J, Bouhouch I, Boutrit C, "Nonlinear minimization algorithm for determining the solar cell parameters with microcomputers," International Journal of Solar Energy., vol. 4, no. 1, pp. 1 12, Jan.1986 